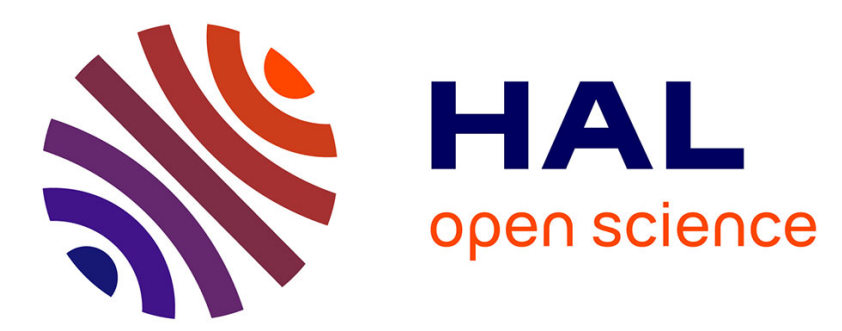

\title{
La théorie de la rumination : état de l'art et perspectives de recherche en comportement du consommateur
}

\author{
Alain Debenedetti, Pierrick Gomez
}

\section{To cite this version:}

Alain Debenedetti, Pierrick Gomez. La théorie de la rumination: état de l'art et perspectives de recherche en comportement du consommateur. Recherche et Applications en Marketing (French Edition), 2006, 21 (1), pp.41-55. hal-01214653

\section{HAL Id: hal-01214653 \\ https://hal.science/hal-01214653}

Submitted on 12 Oct 2015

HAL is a multi-disciplinary open access archive for the deposit and dissemination of scientific research documents, whether they are published or not. The documents may come from teaching and research institutions in France or abroad, or from public or private research centers.
L'archive ouverte pluridisciplinaire HAL, est destinée au dépôt et à la diffusion de documents scientifiques de niveau recherche, publiés ou non, émanant des établissements d'enseignement et de recherche français ou étrangers, des laboratoires publics ou privés. 
La théorie de la rumination: état de l'art et perspectives de recherche en comportement du consommateur

\section{Alain Debenedetti}

Attaché Temporaire d'Enseignement et Recherche à l'Université Paris-Dauphine, centre de recherche DMSP, Place du Maréchal de Lattre de Tassigny, 75775 Paris cedex 16,

Doctorant à l'ESSEC

Tel : 01.44.05.44.59

Mail : alain.debenedetti@ dauphine.fr

53, avenue Parmentier, 75011 Paris

Tel : $0148072163-$ Mob : 0672077979

\section{Pierrick Gomez}

Doctorant à l'Université de Nantes, LAGON (Université de Nantes) et LARGECIA (Ecole Nationale des Techniques des Industries Alimentaires et Agricoles), Rue de la Géraudière BP 82225, 44322 Nantes Cedex 03

Tel : 02.51.78.54.54

Mail : pierrick.gomez@wanadoo.fr

Appartement B31, 5-7 rue Donatello, 92400 Courbevoie

Tel : 0147891674 - Mob : 0684373110 
La théorie de la rumination: état de l'art et perspectives de recherche en comportement du consommateur

\section{RESUME}

Martin et Tesser $(1989,1996 \mathrm{a})$ ont proposé une «théorie de la rumination » pour décrire les comportements des individus qui, sans véritable justification, ressassent des pensées négatives. Leur approche motivationnelle fait de la rumination une forme de pensée souvent contre-productive relative à un décalage entre un objectif poursuivi et un état actuel. Cette théorie prometteuse a fait l'objet de nombreux débats en psychologie clinique mais d'aucun développement en comportement du consommateur. Cet article synthétise de manière critique les travaux sur les pensées ruminatives et propose des pistes de recherche en marketing. Mot clés : rumination, comportement du consommateur, processus de décision

Rumination Theory: Literature Review and Avenues for Research in Consumer Behavior

\section{SUMMARY}

Martin and Tesser $(1989,1996)$ proposed a "rumination theory" to describe a form of behavior where individuals dwell on recurrent negative thinking for no particular reason. Adopting a motivational approach, they present rumination as an often counterproductive thinking which stems from a discrepancy between pursued goal and current state. However, while this promising theory has received substantial attention in clinical psychology, it has to date not been tackled in the literature on consumer behavior. Thus, this paper aims first at synthesizing the current body of research on ruminative thoughts and second at suggesting directions for research in marketing.

Key words : rumination, consumer behavior, decision making process 
La théorie de la rumination : état de l'art et perspectives de recherche en comportement du consommateur

Tourner et retourner quelque chose dans son esprit sans pouvoir s'en empêcher : c'est bien de cela qu'il s'agit lorsqu'on évoque la notion de rumination. Ensemble de pensées récurrentes, persistantes, souvent intrusives et contre-productives, la rumination a d'abord été évoquée dans les recherches sur les conséquences d'évènements traumatiques (Horowitz, 1976 ; Janoff-Bulman, 1992 ; Nolen-Hoeksema et Morrow, 1991 ; Silver et Wortman, 1980 ; Tait et Silver, 1989) et l'explication de certains désordres mentaux (Davis et NolenHoeksema, 2000 ; Lyubomirsky et al., 1999 ; Nolen-Hoeksema, 1996 ; Rachman, 1991 ; Segerstrom et al., 2000 ; Van Rijsoort, Emmelkamp et Vervaeke, 2001). Des recherches ont ensuite sorti la rumination de son contexte clinique, en particulier la «théorie de la rumination » de Martin et Tesser (1989, 1996a), qui représente la première tentative d'intégration de la rumination dans un cadre théorique large, ancré dans la vie courante.

L'approche retenue par Martin et Tesser peut être inscrite dans le cadre des travaux sur les motivations et les volitions. Cette approche scinde les processus motivationnels en deux étapes distinctes qui se suivent chronologiquement (Heckhausen, 1991 ; Gollwitzer, 1990). La première étape, pré-décisionnelle, voit les buts se former alors que la deuxième étape, postdécisionnelle (appelée aussi volitionnelle), consiste en la mise en œuvre des intentions préalablement formées. Cette dernière étape implique de planifier, contrôler et maintenir les comportements afin de les garder en ligne avec les intentions. Dans ce cadre, la rumination apparaît dans la phase volitionnelle et plus particulièrement de contrôle : elle survient lorsque les performances ne sont pas à la hauteur des intentions.

Si la rumination n'a fait l'objet d'aucune recherche en marketing à la connaissance des auteurs, dans la vie d'un consommateur, les occasions de ruminer sont multiples. Lors du 
processus d'achat, un consommateur peut par exemple se sentir démuni face à une offre pléthorique ou pour laquelle il ne se sent pas expert. Il risque alors de se focaliser sur ce but «menaçant» et d'être incapable de s'en détourner, ce qui peut se traduire par un report d'achat. Le consommateur peut également se focaliser durablement sur un but de consommation qui n'a pas été atteint, alors synonyme de regret, de déception ou d'embarras. Cet échec peut alimenter le sentiment d'insatisfaction né de l'achat incriminé. Ces illustrations, qui soulignent l'aspect négatif de la rumination, ancrent le concept dans le processus de décision d'achat avec comme questions sous-jacentes : Quel est le rôle de la rumination dans les différentes étapes du processus de décision? Quel statut revêt alors cette variable : modère-t-elle le lien entre différentes variables intervenant dans le processus de décision; ou bien doit-on considérer que certaines conséquences de l'état émotionnel du consommateur ne surviennent que dans la mesure où le processus de rumination s'est enclenché ? L'intérêt d'introduire la rumination dans la sphère du comportement du consommateur réside également dans la diversité des phénomènes où elle intervient. A travers leur approche motivationnelle, Martin et Tesser (1996a) envisagent également l'existence de pensées récurrentes positives et liées à l'atteinte d'un but. Certaines techniques de communication pourraient alimenter une rumination positive. Le problème qui se pose alors pour les managers est de faire en sorte que le consommateur rumine des pensées agréables à propos d'un produit ou d'une marque à venir. Enfin, si nos illustrations de la rumination dans le champ du comportement du consommateur présentent la rumination comme une activité cognitive qui fait suite à un décalage entre une situation vécue et un but visé, un certain nombre de recherches en psychologie clinique envisagent plutôt la rumination comme un trait de personnalité (Scott et McIntosh, 1999 ; Sukhodolsky, Golub et Cromwell, 2001). Ces travaux nous amènent à nous poser les questions suivantes : existe-t-il des consommateurs 
davantage susceptibles de s'engager dans un processus de rumination que d'autres ? Ont-ils un profil particulier?

L'intégration de la rumination dans le champ du comportement du consommateur permet d'envisager de nombreux développements. Si les approches cognitives et socio-cognitives de la rumination sont intéressantes, nous avons choisi d'axer notre article autour de l'approche motivationnelle de Martin et Tesser (1989, 1996a), particulièrement adaptée pour mieux comprendre les perturbations du processus de décision d'achat du consommateur. Dans cette optique, nous présenterons de manière critique le concept de rumination de Martin et Tesser en comparaison aux autres modèles traitant des pensées récurrentes, puis nous en préciserons les modalités et les mécanismes en les inscrivant dans une perspective marketing. Enfin, nous détaillerons les principales pistes de recherche qu'il nous paraît pertinent de souligner dans le cadre d'une meilleure compréhension des comportements de consommation.

\section{QU'EST CE QUE LA RUMINATION MENTALE ?}

La «théorie de la rumination » (Martin et Tesser, 1989, 1996a) trouve son origine dans les travaux de Zeigarnik (1938), qui découvrit que les individus se souvenaient mieux des tâches inachevées que des tâches effectuées complètement. Ces recherches montraient entre autres que ce phénomène dépendait de l'importance de l'objectif, du chemin à effectuer pour l'atteindre, de sa clarté et de la présence d'objectifs substituables. L'approche motivationnelle de Martin et Tesser n'est bien sûr pas la seule manière d'envisager la rumination. D'autres théories, d'origine cognitive, s'y intéressent. Si elles offrent des perspectives plus limitées en comportement du consommateur que le principe d'incomplétude à laquelle les recherches de Zeigarnik (1938) font référence, ces modèles permettent de replacer les travaux de Martin et Tesser $(1989,1996 a)$ dans le cadre plus global des réponses 
à un épisode émotionnel. Nous commencerons donc par brièvement présenter ces théories, dont le point commun est d'ancrer la rumination dans les processus de réponse aux émotions.

Les théories non motivationnelles de la rumination : la rumination comme traitement cognitif des émotions

Depuis une trentaine d'années, la rumination mentale fait l'objet de recherches en psychologie, où elle est le plus souvent considérée comme un traitement cognitif de l'émotion. Cette réévocation mentale des évènements, comme la communication des évènements à l'entourage social (appelée partage social de l'émotion ${ }^{1}$ ), constituent les conséquences cognitives et sociales à long terme d'un épisode émotionnel, indépendamment de la nature de l'événement émotionnel, de sa valence ou du type d'émotion évoqué (Rimé et al., 1992). Etudiée dans le cadre des conséquences intrapersonnelles des épisodes émotionnels (le partage de l'émotion constituant la réponse sociale, interpersonnelle), la rumination est considérée à court terme comme une réponse adaptative à une situation émotionnelle. En revanche, sa persistance à long terme est synonyme de mauvaise adaptation psychologique.

D’abord envisagée en lien avec les évènements traumatiques, la rumination indique le niveau de traitement mental de l'information émotionnelle relative au trauma, avec des mécanismes cognitifs différents: processus de réévaluation de l'événement en vue de restaurer une maîtrise sur l'apparition des pensées (Horowitz, 1976), de reconstruction des croyances de bases ébranlées par le trauma (Janoff-Bulman, 1992) ou de recherche de signification dans l'approche socio-cognitive de Tait et Silver (1989). Dans cette dernière, un soutien social adapté permet également de reprendre contrôle sur l'environnement et contribue à sortir du processus de rumination (Luminet, 2002).

\footnotetext{
${ }^{1}$ Pour plus de détail, notamment sur la propension des individus à communiquer avec leur entourage à propos des évènements émotionnels, cf. Luminet, 2002.
} 
Les recherches effectuées dans le contexte des troubles obsessionnels (Rachman, 1991) et de la dépression (Nolen-Hoeksema, 1996) constituent le premier pas vers l'élargissement du champ de la rumination, notamment en comparant patients non cliniques et patients atteints de pathologies. Dans l'approche de Rachman (1981), si tout le monde connaît des pensées ruminatives, les patients cliniques les vivent plus fréquemment, plus intensément et ont davantage de mal à s'en défaire. Comme dans le modèle de Nolen-Hoeksema, où la rumination (souvenirs liés à l'événement déclencheur) est un mode de réponse à la dépression au même titre que la distraction, les pensées intrusives sont essentiellement dysfonctionnelles.

Plus récemment, Wegner (1992) s'est s'intéressé aux réactions individuelles consécutives à l'apparition de pensées perçues comme désagréables. Les individus chercheraient à supprimer les pensées non désirées, afin de ne pas ressentir les émotions négatives qui leur sont liées. Ces stratégies d'évitement auraient une conséquence paradoxale, appelé « effet de rebond»: la tentative de suppression serait suivie par un retour en mémoire plus fréquent de l'événement qu'avant. Si toutes ces approches font de la rumination un indicateur du degré de profondeur du traitement cognitif de l'émotion, le modèle de Martin et Tesser (1989, 1996a), fondé sur une approche motivationnelle, fait exception.

\section{La rumination dans l'approche motivationnelle de Martin et Tesser}

\section{Définition de la rumination}

Martin et Tesser $(1989,1996 a)$ ancrent leurs recherches délibérément dans la vie quotidienne, hors du champ des pensées récurrentes liées à un traumatisme. Loin du mode de traitement de l'information émotionnelle relative aux traumas de Horowitz (1976), Martin et Tesser définissent la rumination (1996a, p1) comme «une classe de pensées conscientes, concernant un thème banal et instrumental, récurrentes même en l'absence d'un 
environnement immédiat prédisposant à l'activation de ces pensées ». Cette définition a été critiquée par les tenants d'une approche dysphorique de la rumination car elle fait du construit une notion un peu trop large (Nolen-Hoeksema, 1996). En dépit des commentaires suscités, cette définition constitue cependant une vision pragmatique de la rumination (Wänke et Schmid, 1996) puisqu'elle synthétise les visions précédentes, les simplifie et permet d'établir un pont entre la psychologie clinique et la psychologie sociale. Elle permet d'intégrer d'autres perspectives, comme par exemple celle de Tait et Silver (1989), où la rumination est vue comme un ensemble de pensées, de souvenirs et d'images mentales liés à l'événement émotionnel, ou celle de Janoff-Bulman (1992), qui la définit comme un ensemble de pensées involontaires et perturbantes apparaissant de manière récurrente. Ainsi, la principale faiblesse de la théorie de la rumination constitue aussi sa principale force : permettre d'élargir le champ d'application du concept. Deux aspects majeurs apparaissent dans la définition de Martin et Tesser: d'abord que la rumination est une forme de pensées récurrentes ; ensuite, qu'elle concerne des pensées instrumentales, c'est à dire orientées vers des buts.

\section{Des pensées récurrentes en réponse à l'interruption des buts}

Ce qui caractérise d'abord la rumination, c'est la récurrence des pensées. La rumination ne se distingue pas des autres pensées par son contenu, son intensité ou par la spécificité de l'activité mentale qu'elle implique, mais par la circularité du processus (Wänke et Schmid, 1996). Elle est donc à distinguer des pensées éphémères ou des pensées furtives dirigées vers des buts transitoires (Martin et Tesser, 1996a). Si certains contextes peuvent favoriser leur développement, les pensées ruminatives sont incontrôlées puisqu'elles ont tendance à revenir même en l'absence d'un environnement susceptible de les réactiver (Koole et al., 1999).

La théorie de la rumination de Martin et Tesser (1989, 1996a) part du principe que l'activité mentale est instrumentalisée, c'est-à-dire qu'une pensée est toujours le fruit de la 
volonté d'atteindre un but. Il est en effet aujourd'hui admis que l'existence de buts permet aux individus de donner du sens à leur vie et guide de nombreux comportements (Austin et Vancouver, 1996 ; Carver et Scheier, 2000 ; Baumeister, 1991). La rumination n’échappe pas à ce principe. S'inspirant des modèles d'autorégulation dit «cybernétiques » (Carver et Scheier, 1981, 2000), Martin et Tesser partent du principe que les individus comparent constamment les buts poursuivis aux performances obtenues afin d'ajuster leurs comportements en fonction. Lorsque ces buts ne peuvent être atteints ou lorsque le rythme de progrès est jugé insatisfaisant par l'individu, la rumination est susceptible d'apparaître. L'interruption des buts peut alors contribuer au maintien de la rumination (Martin et Tesser, 1996a, Scott et McIntosh, 1999). Ces buts peuvent concerner la consommation, comme acheter une maison, arrêter de fumer (Bagozzi et Warshaw, 1990) ou bien offrir un cadeau (Huffman, Ratneshwar et Mick, 2000). Toute interruption d'un but est susceptible de conduire à la rumination mentale, mais l'importance du but détermine la durée et sa fréquence de la rumination. L'approche retenue par Martin et Tesser (1996a) se rapproche de celle proposée par Kuhl (1994). En effet, pour celui-ci la rumination intervient dans une phase de la poursuite des buts qu'il appelle «l'orientation-attente»(state orientation). Lors de cette phase, l'individu concentre son attention sur ses succès passés ou futurs afin d'analyser les causes et conséquences d'un état interne (humeur...) ou d'un état externe (problème...).

Par ailleurs, la rumination peut concerner plusieurs sortes de buts. En effet, les objectifs des individus sont structurés hiérarchiquement et composés de buts mineurs (plutôt concrets : acheter un tube de colle pour réparer un objet cassé) et de buts majeurs (plutôt abstraits : réussir sa vie) définis de manière subjective (Martin et Tesser, 1989, 1996a; NolenHoeksema, 1996), l'individu poursuivant rarement un seul but à la fois. L'individu n'est pas nécessairement conscient de tous les niveaux hiérarchiques: seule l'apparition d'une difficulté permet d'identifier les objectifs d'ordre supérieur. Ainsi, la hiérarchie des buts 
expliquerait pourquoi la rumination survient parfois pour des raisons en apparence futiles. Elle révèlerait une incapacité à identifier le but d'ordre supérieur couvert par le but, hiérarchiquement inférieur, menacé. Enfin, si la théorie de Martin et Tesser reste floue à cet égard, il est probable que la rumination s'enclenche plus facilement dans le cadre de la poursuite de buts relevant de l'identité de l'individu. En effet, il est admis que les buts et leur gestion relèvent d'une des fonctions majeures du soi (Carver et Scheier, 2000).

\section{Différentes formes de rumination?}

Dans l'approche de Martin et Tesser (1989, 1996a), la rumination comporte 3 dimensions :

- La valence : la rumination peut revêtir une forme négative (par exemple : regretter un achat effectué) ou positive (par exemple : anticiper la sortie d'une console de jeu vidéo) ;

- L'orientation temporelle de la pensée : la rumination peut concerner un évènement passé, présent ou futur. Un consommateur peut en effet ruminer sa déception vis à vis d'un produit qu'il vient d'acquérir ou anticiper la diffusion de la coupe du monde de rugby sur la chaîne câblée à laquelle il vient de s'abonner ;

- Le type de polarisation ou niveau de complétude de la pensée : la rumination peut concerner un but non atteint (par exemple, être déçu d'avoir raté les soldes) ou bien reposer sur l'absence de progression perçue quant à l'atteinte de ce but (par exemple, s'inquiéter de ne pas obtenir un prêt pour l'achat d'un appartement).

Cette classification de la rumination témoigne de la volonté des auteurs d'élargir le champ de la rumination à la vie courante mais montre cependant des limites : modes non décrits car ne se produisant jamais; modes utilisant des termes imprécis, difficilement comparables ou contradictoires ; enfin, inclusion du temps présent, en décalage avec l'idée de récurrence des pensées sur «longue période de temps » présentée par les chercheurs eux- 
mêmes (Martin et Tesser, 1996a, p12). Roger et Najarian (1989) limitent d'ailleurs la rumination à un type de pensée ne s'inscrivant que par rapport à des évènements passés ou futurs.

\section{Un processus dynamique traduisant un échec du «coping »?}

La rumination s'inscrit dans un processus dynamique qui s'engage lorsque l'individu perçoit une frustration relative à un but de niveau important. Elle apparaît après un premier épisode de répression des pensées (Martin, Tesser et Mc Intosh, 1993). Pour stopper le cycle de rumination, seule l'atteinte du but ou son abandon s'avèrent efficaces. Les autres routes (répétition de la même réponse avec une intensité supérieure, recherche de solutions alternatives, renégociation de l'objectif à atteindre ou engagement dans une phase de distraction éphémère) tendent à conduire à la poursuite du cycle. A mesure que les épisodes du cycle s'enchaînent, des éléments de contexte et des associations entre le contenu des pensées ruminatives et des pensées qui partagent des similarités de sens (Luminet, 2002) viennent renforcer la rumination. La rumination change alors de contenu : l'individu se focalise sur les sentiments associés à son échec et met de côté les pensées relatives aux moyens à mettre en œuvre pour atteindre le but. L'individu risque alors de sombrer dans la dépression. Ce processus rappelle par certains aspects les stratégies de «coping ». Heim (1995) voit d'ailleurs dans la rumination l'échec d'une stratégie de « coping ». La rumination constitue alors une phase terminale, qui intervient lorsque toutes les tentatives de résolution de problème ou d'évitement ont échoué et que l'individu n'arrive plus à faire face.

Les différentes approches de la rumination étant mises en perspective, il s'agit à présent de préciser les mécanismes qui gouvernent l'apparition de telles pensées récurrentes. Là encore, nous partirons des propositions faites par Martin et Tesser (1989, 1996a). 


\section{Antécédents de la rumination}

Qu'est ce qui pousse l'individu à ruminer? En proposant la non atteinte d'un but perçu comme important par l'individu comme instigateur de la rumination, Martin et Tesser (1996a) font de ce construit un phénomène d'origine motivationnelle. En effet, l'identification d'un décalage entre un état actuel et un état souhaité, et l'impossibilité de mettre en œuvre une stratégie de réduction de ce décalage sont fréquemment évoqués pour expliquer le phénomène (Wänke et Schmid, 1996). Si l'origine cognitive de la rumination est prépondérante, le rôle des émotions ne doit pour autant pas être minoré. Ainsi, Luminet et al. (2000) trouvent une relation positive entre niveau d'intensité émotionnelle (engendré par un évènement $\mathrm{x}$ ) et rumination mentale. De même, Lyubomirsky et Nolen-Hoeksema (1995) définissent la rumination comme un processus mobilisant l'attention sur ses humeurs. Colère, anxiété ou encore humeurs négatives peuvent être accompagnées de pensées ruminatives (Lyubomirsky et al., 1999).

Cependant si le rôle des émotions est souvent évoqué, c'est bien la détection d'une perturbation dans le processus d'atteinte des buts qui est déterminante dans le déclenchement des pensées récurrentes. En effet, la théorie de «l'appraisal », suggère que le déclenchement et la différenciation des émotions s'effectuent à travers les cognitions. Or, l'évaluation des buts constitue une séquence importante du traitement du stimulus émotionnel (Lazarus et Folkman, 1984). Les individus interprètent alors leur état émotionnel comme un signal pertinent pour juger de leur position par rapport au but poursuivi et des directions à prendre pour les atteindre (Frijda, 1988; Ekman, 1999). Parmi les différentes classes d'émotions, certaines d'entre elles impliquent plus particulièrement l'évaluation de buts liés à l'identité de l'individu (Tracy et Robins, 2004) et pourraient être particulièrement impliquées dans la rumination: on range parmi elle la honte, la culpabilité, l'embarras (Tangney et Fischer, 
1995) ou encore le regret (Verbeke, Belschaft et Bagozzi, 2004). Martin et Tesser (1996a) en concluent qu'un affect ou une humeur négative peuvent être à l'origine de pensées ruminatives mais uniquement dans un contexte de difficulté dans l'atteinte d'un but. L'émotion ne constitue donc pas un antécédent de la rumination mais plutôt une preuve de la pertinence du jugement sur la position face au but à atteindre (Watkins et Mason, 2002).

L'impossibilité d'atteindre un but comme nécessaire déclencheur de la rumination fait néanmoins l'objet de discussions. Certains n'y voient qu'une cause éventuelle (Koole et al., 1999). Wänke et Schmid (1996) indiquent que l'individu rumine parfois sans qu'on puisse établir un lien avec un quelconque but ou à propos de buts qu'il sait pertinemment hors d'atteinte. L'hypothèse est donc émise que c'est la prise de conscience d'un état alternatif (subjectivement supérieur) qui est à l'origine de la rumination (King et Pennebaker, 1996). De même, le sentiment de perte de contrôle du décalage entre la situation actuelle et la situation souhaitée ou l'impossibilité de mettre en œuvre une stratégie de réduction de ce décalage pourraient être à l'origine de la rumination (Wänke et Schmid, 1996). Heim (1995, p148) parle alors de «mauvais coping». Martin et Tesser (1996b) évoquent cette possibilité en posant que c'est l'échec dans la recherche de méthodes alternatives qui est à l'origine de la rumination. Cet échec déclenche alors le passage de la sphère instrumentale (pensée orientée vers les moyens) à la sphère affective (pensée orientée vers les buts). La rumination et son pan émotionnel prennent alors le pas sur la résolution du problème. Enfin, le rôle du contexte doit être également envisagé car il a été montré que les évènements traumatiques, tels que la perte d'un proche ou l'implication dans une catastrophe naturelle peuvent être directement à l'origine de pensées ruminatives (Horowitz, 1976 ; Janoff-Bulman, 1992 ; Rimé et al., 1992). 


\section{Les facteurs influençant la rumination}

Si Roger et al. (2000) démontrent que l'origine ethnique n'a pas d'influence sur la propension à ruminer, Wicklund et Braun (1987) montrent qu'un individu âgé ou disposant de plus d'expérience a davantage de solutions à sa disposition lorsqu'un but est menacé donc qu'il est moins sujet à la rumination; Nolen-Hoeksema (2005) conclut à une plus forte propension des femmes à ruminer. Qu'en est-il des facteurs liés à la personnalité de l'individu ? De nombreux travaux montrent que tous les individus ne sont pas égaux face à la rumination et ont abordé le construit sous l'angle de la tendance à ruminer (Sukhodolsky, Golub et Cromwell, 2001 ; Scott et Mc Intosh, 1999 ; Van Oppen, Hoekstra et Emmelkamp, 1995). On peut donc émettre l'hypothèse, en dehors du fait qu'il existe des occasions propices à la rumination, que certains individus ont davantage tendance que d'autres à entrer dans un cycle de rumination. La littérature propose de nombreux facteurs influençant la rumination (l'estime de soi : Di Paula et Campbell (2002); l'introversion : Nolen-Hoeksema, Parker et Larson, 1994 ; l'anxiété : Mellings et Alden, 1999 ; Nolen-Hoeksema, 2000a). Nous en avons sélectionné un petit nombre nous paraissant pertinents dans l'étude du comportement du consommateur.

Premièrement, la tendance à persister (Davis et Nolen-Hoeksema, 2000) : elle consiste en une incapacité de modifier son comportement lorsqu'une information ou une conséquence nouvelles apparaissent ou lors d'une modification de l'environnement. Elle entraine une difficulté à générer de nouvelles alternatives et à se détourner d'un problème : un lien a été montré entre tendance à ruminer et tendance à persister (Davis et Nolen-Hoeksema, 2000) : les « ruminateurs » semblent donc différer des autres individus dans leur manière de (ne pas) rechercher et traiter l'information. Deuxièmement, le « locus » de contrôle (Wänke et Schmid, 1996) : il oppose les individus qui ont tendance à s'attribuer les succès ou les échecs d'une situation (« locus » de contrôle interne) et les individus qui les attribuent à des forces externes, 
comme le contexte ou le hasard (« locus » de contrôle externe). Ainsi, les personnes ayant un « locus » de contrôle interne semblent devoir souffrir davantage des évènements négatifs qui leur arrivent, comme la constatation d'un écart négatif important entre leur objectif personnel et leur état actuel. Une expérimentation réalisée par Wänke et Schmid (1996) a effectivement montré l'existence d'une relation entre «locus» de contrôle interne et rumination. Troisièmement, le neuroticisme : défini comme la tendance à ressentir des émotions négatives telles que l'anxiété ou la détresse (Mc Rae et John, 1992), il est souvent relié à la rumination (Trappnell et Campbell, 1999 ; Luminet et al., 2000). On peut donc s'attendre à ce que les individus présentant un score élevé sur la dimension neuroticisme du modèle en 5 facteurs entrent plus facilement dans un cycle de pensées récurrentes négatives. Enfin, l'orientation de la pensée : d'après la théorie de Kuhl $(1981,1994)$, les individus dont la pensée est orientée vers l'analyse (orientation-attente) sont moins capables de générer des actions efficaces pour résoudre leurs problèmes, d'abandonner un but qu'ils considèrent ne pas pouvoir atteindre, de se distraire ou de changer d'objectif que les individus dont la pensée est orientée vers l'action (orientation-action). Ils seraient donc davantage sujets à la rumination.

\section{Les conséquences de la rumination}

La rumination affecte de façon concomitante le système cognitif et affectif pouvant perturber le processus de prise de décision. Plusieurs types de conséquences sont mises en évidence dans la littérature. Premièrement, une accentuation de l'intensité et de la durabilité des émotions ressenties (Bushman, 2002 ; Martin et Tesser, 1989; Rusting et NolenHoeksema, 1998 ), avec une association à des expériences prolongées et intenses d'humeurs négatives (Lyubomirsky et Nolen-Hoeksema, 1995 ; Nolen-Hoeksema et Morrow, 1993), plus particulièrement de tristesse (Nolen-Hoeksema et Morrow, 1991). Dans les cas extrêmes, la rumination peut être à l'origine de dépression et symptômes anxieux (Martin et Tesser, 
1996a ; Nolen-Hoeksema, 1996). Deuxièmement, la rumination entraîne un déclin des performances cognitives (Scott et Mc Intosh, 1999, Siegle et al., 2003). L'individu voit baisser sa capacité à résoudre les problèmes ainsi que son attention (Watkins et Baracaia, 2002 ; Sukhodolsky, Golub et Cromwell, 2001) car un manque de motivation à agir et une humeur négative interviennent. L'individu perd alors confiance en ses capacités à atteindre son but (Lyubomirsky et al., 1999). Il arrive même qu'il soit conscient des solutions à sa disposition mais qu'il ne puisse pas les mettre en œuvre. Troisièmement, la rumination provoque des biais de jugement négatif tendant à fausser le choix de l'individu qui rumine et à rendre les problèmes plus menaçants qu'ils ne le sont (Lyubomirsky et al., 1999). Les pensées ruminatives provoquent également des biais informationnels : la rumination conduirait à une préférence mémorielle pour les informations négatives (Williams et Oaksford, 1992) et pourrait encourager l'individu à rechercher l'ensemble des informations négatives rattachées à la sensation qu'il expérimente (Lyubomirsky et al., 1999). Par ailleurs, la rumination étant un processus d'exploration de ses sentiments ou pensées (Trappnell et Campbell, 1999), l'individu est susceptible d'opter pour une recherche d'information interne. Il s'ensuivrait un besoin de réassurance excessif (Nolen-Hoeksema, 2000b). Quatrièmement, la rumination est associée à un besoin de ressources temporelles supérieur à la moyenne en phase de prise de décision (Nolen-Hoeksema, 2000b). Ward et al. (2003) montrent que les personnes qui ruminent ont besoin de plus de temps que les autres pour se sentir satisfaits de leurs décisions. Elle serait alors marquée par une forte hésitation à agir, qui naîtrait de leur manque de confiance et de satisfaction à l'égard des plans qu'ils échafaudent afin de résoudre leurs problèmes. En effet, dans la mesure où elle interfère avec les processus de motivation conduisant à l'action, la rumination conduit l'individu à l'inertie et l'empêche de se tourner vers l'essentiel : la résolution du problème (Kuhl, 1994 ; Lyubomirsky et Tkach, 2003). 


\section{Les mécanismes de cessation de la rumination}

De nombreux auteurs s'accordent sur les raisons principales permettant un arrêt du phénomène de rumination (King et Pennebaker, 1996 ; Koole et al., 1999 ; Martin et Tesser, 1996a; Wänke et Schmid, 1996). Quatre mécanismes sont identifiés : l'atteinte du but, le désengagement lorsque l'écart constaté n'est plus considéré comme pertinent, le changement d'objectif ou la distraction. A noter que ce dernier mécanisme, s'il est efficace, ne permet qu'un arrêt temporaire de la rumination (Wänke et Schmid, 1996 ; Koole et al., 1999) et comporte des risques tels que l'accroissement de la saillance et de l'accessibilité cognitive de la pensée non désirée (Wegner et al., 1987) et en retour l'accroissement de l'intensité des pensées ruminatives (Gold et Wegner, 1995). D’autres mécanismes de gestion émergent dans la littérature. D'abord, l'affirmation de soi, qui conduit à un affect positif et réduit l'accessibilité aux informations liées à la non atteinte du but, donc aux pensées ruminatives (Koole et al., 1999). Ensuite, la capacité à communiquer : King et Pennebaker (1996) ont montré que parler ou écrire à propos d'un traumatisme semblait permettre d'effectuer une « rumination productive ». Les individus qui ont facilement tendance à communiquer auraient donc moins tendance à ruminer. Dans un contexte marketing, c'est en particulier à ces deux derniers mécanismes de gestion que le manager va devoir s’intéresser : comment redonner de la confiance au consommateur et lui permettre de s'affirmer? Comment lui donner l'occasion de s'exprimer à propos de ses buts contrariés?

\section{PERSPECTIVES DE RECHERCHE EN COMPORTEMENT DU CONSOMMATEUR}

Dans cette partie, nous allons d'abord montrer comment l'approche motivationnelle de Martin et Tesser $(1989,1996 a)$ permet d'intégrer la théorie de la rumination dans l'étude du comportement du consommateur, en particulier comment la rumination négative intervient 
lorsque le processus d'achat est perturbé. Nous soulignerons les différentes situations propices à l'apparition de la rumination et les différents rôles que ce phénomène peut jouer dans le processus de décision du consommateur. Nous discuterons ensuite l'intérêt d'envisager la rumination comme une variable individuelle. Enfin nous réfléchirons aux pistes de recherche concernant la rumination positive, notamment dans le domaine de la communication.

\section{La rumination : élément de perturbation du processus de décision d'achat?}

Lors du processus d'achat, à quel moment la rumination est-elle susceptible d'intervenir? Quels sont ses effets sur le consommateur? La littérature montre que mobiliser le concept de rumination dans le cadre des perturbations du processus d'achat s'avère pertinent. En effet, Alderson (1957) ou plus récemment Huffman, Ratneshwar et Mick (2000) envisagent l'achat comme un acte orienté vers des buts et qui nécessite de résoudre des problèmes (liés à l'information sur les produits et les marques, le prix, l'expertise dans la catégorie de produits etc.) : le consommateur poursuit un but au travers de ses achats et au cours du processus de décision et les occasions de les voir bloqués, ralentis ou paraissant soudainement hors de portée sont multiples. On retrouve à travers cette vision de l'achat l'aspect instrumental des pensées ruminatives. La rumination comporte par ailleurs une dimension volitionnelle. La littérature sur les volitions nous apprend qu'avoir l'intention de poursuivre un but ne garantit pas de l'atteindre, la phase d'opérationnalisation des intentions (nécessitant de sélectionner des moyens et des stratégies, de planifier l'action, de contrôler et réguler ses comportements) jouant un rôle particulièrement critique dans la mise en œuvre des comportements d'achat (Bagozzi, 1993). En intervenant dans une phase critique des processus motivationnels, la rumination peut alors venir perturber le déroulement normal du processus de décision et modifier la réponse comportementale du consommateur. Ainsi, envisager la rumination dans les études sur le comportement du consommateur peut aider à mieux comprendre le lien entre intention et comportement. De plus, les différentes formes de rumination identifiées dans 
l'approche motivationnelle de Martin et Tesser (1996a) revêtent un sens dans le contexte du processus de décision d'achat, de par leur aspect temporel et leur niveau de complétude. Cette perspective dimensionnelle permet également de mieux comprendre quelles peuvent être les contextes d'achat générateurs de pensées négatives (regret, frustration...) et leurs conséquences pour le consommateur. En effet, les situations d'achat génératrices de pensées récurrentes négatives semblent intervenir plus souvent que les situations qui provoquent des pensées ruminatives positives. Cette focalisation sur la rumination négative est d'autant plus pertinente que les évènements négatifs ont des conséquences émotionnelles et comportementales plus intenses pour l'individu que les évènements positifs (Baumeister et al., 2001). A quel moment, dans ces conditions, les pensées ruminatives sont-elles susceptibles d'apparaître?

L'intervention de la rumination dans le processus d'achat: quand et pour quelles raisons?

Avant l'achat, dans un contexte de reconnaissance du problème de consommation, de recherche d'information et d'évaluation des possibilités, qu'est ce qui peut conduire le consommateur à penser qu'il est loin de son but ou qu'il ne peut pas l'atteindre ? Martin et Tesser (1996a) ont proposé que l'inquiétude (Worry) soit le principal mode de rumination dans cette situation. Dans le cadre du processus d'achat, on peut émettre l'hypothèse que l'apparition de pensées récurrentes chargées d'inquiétude soit liée au risque perçu ${ }^{2}$ relatif à l'achat. En effet, les alternatives de choix peuvent être caractérisées par l'incertitude qu'elles impliquent («Est ce que la marque choisie sera aussi satisfaisante que la marque actuelle ?»), par la perte potentielle qui leur est liée (« Est ce que je n’achète pas un produit qui sera bientôt beaucoup moins cher?») ou les deux. Cox (1967) intègre dans la composante de perte les

\footnotetext{
${ }^{2}$ Rappelons que le risque perçu met en jeu « la perception d'une incertitude relative aux conséquences négatives potentiellement associées à une alternative de choix »(Volle, 1995, p39).
} 
buts visés mais non atteints, ce qui correspond aux origines de la rumination dans l'approche motivationnelle.

En aval de l'achat, la phase finale du processus de décision prend la forme d'une évaluation. Lorsque l'achat est source d'insatisfaction, la décision d'achat peut provoquer une variété de réponses affectives qui influencent le contenu des pensées récurrentes. Il est donc pertinent, dans le cadre du processus de décision du consommateur, de proposer l'insatisfaction comme facteur déclenchant la rumination. Comme précédemment, l'insatisfaction sera liée à des formes de pensées récurrentes spécifiques. Si Martin et Tesser (1996a) citent le regret comme mode principal de rumination à propos d'un événement passé, les pensées ruminatives peuvent également être associées à d'autres émotions signalant la menace d'un but important pour le consommateur. Ainsi, une décision malheureuse pourra être source de culpabilité, si l'achat est jugé transgresser une norme (Burnett et Lunsford, 1994) ou de honte, s'il va jusqu'à altérer la perception du soi du consommateur (Tangney et Fischer, 1995), qui en retour alimenteront la rumination.

\section{La rumination : une variable qui revêt différents statuts lors du processus d'achat}

Au-delà du moment où la rumination intervient dans le processus d'achat (en amont ou en aval), et de la nature de ses conséquences (affectives, cognitives ou comportementales), la question du statut théorique de la variable se pose : il est probable que la rumination puisse jouer différents rôles dans le processus d'achat. D'abord, la rumination peut avoir une influence directe sur certaines perturbations du processus de décision d'achat (elle joue alors un rôle médiateur). Ensuite, elle peut interagir avec d'autres variables et modifier certaines conséquences du processus de décision (elle joue alors un rôle modérateur). Enfin, comme le soulignent Wänke et Schmid (1996), elle ne peut constituer qu'un épiphénomène : une émotion implique la modification du processus d'achat mais celle-ci aurait été identique que 
la rumination apparaisse ou non. Ce dernier cas, qui ne présente pas d'intérêt dans une perspective marketing, ne sera donc pas traité.

Lorsque certaines conséquences d'un événement émotionnel ne se produisent que dans la mesure où la rumination intervient, cette dernière joue un rôle médiateur. Par exemple, lorsque la rumination apparaît lors de la phase de reconnaissance de problème, le consommateur va voir ses capacités cognitives diminuer. Cela peut se traduire par un sentiment d'incapacité de choisir le bon type de réponse à son problème de consommation : «Suis-je capable de déterminer mes besoins en termes de connexion Internet et de téléphonie pour ma maison ? ». La rumination peut alors diminuer l'attention et la motivation à agir du consommateur. Ce dysfonctionnement lié à l'apparition de la rumination peut être renforcé par des biais de jugements négatifs (Lyubomirsky et al., 1999) rendant le problème de consommation plus menaçant qu'il n'est réellement et contribuant également à fausser le choix. Ensuite, lorsque le consommateur recherche de l'information, il est probable qu'il se focalise sur, voire recherche les signaux négatifs des options présentées et mette ainsi à mal l'accomplissement du choix. En effet, la rumination induit aussi une préférence cognitive pour les informations négatives (Williams et Oaksford, 1992), elle-même susceptible d'intensifier le risque perçu. Par ailleurs, l'augmentation de la charge cognitive pourrait influencer la décision du consommateur de différentes manières : d'abord, elle encourage le consommateur à user d'heuristiques de choix simplifiés (Luce, Bettman et Payne, 1999). L'effort cognitif induit par l'acte d'achat orienterait alors le choix du consommateur vers des alternatives simples (par exemple, un choix parmi un ensemble restreint d'alternatives, toujours le même choix ou le choix le moins complexe, par peur de se tromper) en particulier dans un contexte de ressources temporelles limitées (Garbarino et Edell, 1997). Ensuite, la rumination pourrait expliquer un report d'achat soudain puisqu'elle rend l'achat plus difficile par la forte mobilisation des ressources attentionnelles et cognitives, le consommateur devant 
à la fois porter son attention sur le flot de pensées intrusives et effectuer son choix. Or, lorsque l'acte d'achat est perçu comme désagréable, les consommateur ont plus tendance à le reporter (Darpy, 2002). La Figure 1 rend compte du rôle médiateur de la rumination dans le processus d'achat. Elle permet de proposer la rumination comme élément de perturbation du processus d'achat, élément qui peut amener le consommateur à plus facilement faire un « mauvais choix », un «non-choix » ou à adopter des comportements d'achat stéréotypés.

\section{$\underline{\text { Figure } 1 \text { : le rôle médiateur de la rumination }}$}

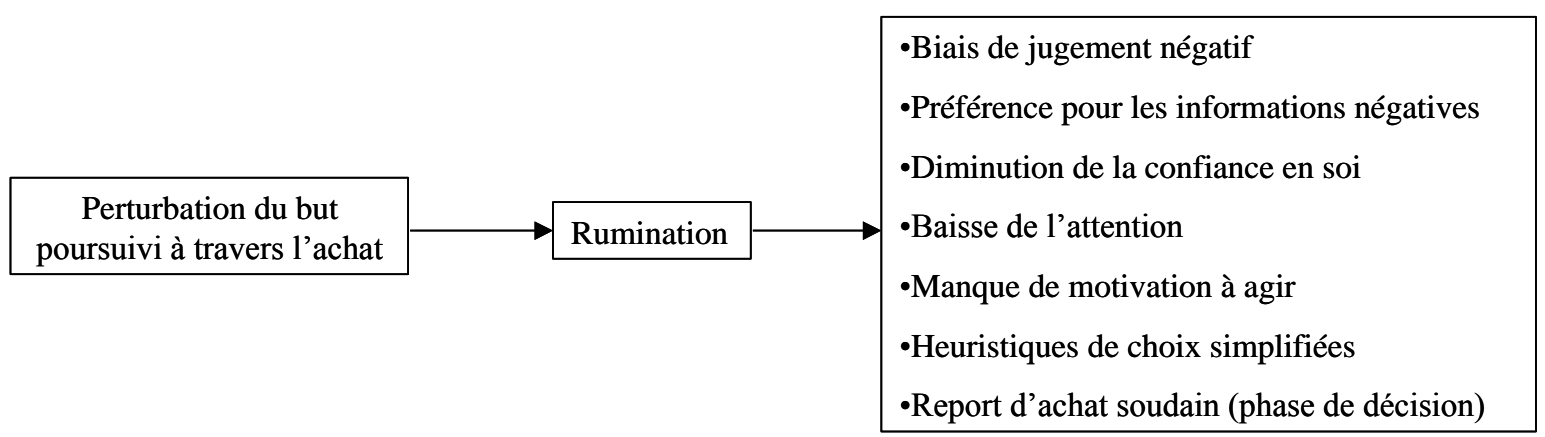

Lorsque la rumination interagit avec d'autres variables et modifie certaines conséquences du processus de décision (et si elle ne constitue pas uniquement une différence individuelle), elle contribue également à enrichir la compréhension de la perturbation de certains processus de décision d'achat. Ainsi, lorsque le consommateur évalue les alternatives de choix, le biais de jugement négatif déjà évoqué est susceptible d'être accompagné d'émotions, comme l'anxiété : («Je serai une mauvaise mère si je choisis un produit qui rend mes enfants obèses »), renforçant l'intensité et la durée de la rumination et des émotions qui y sont associées. Une fois l'achat réalisé et jugé suffisamment insatisfaisant pour entraîner la rumination, la littérature montre qu'il s'ensuit une accentuation et un prolongement des humeurs négatives (Lyubomirsky et Nolen-Hoeksema, 1995 ; Nolen-Hoeksema et Morrow, 1993) pouvant conduire à un rejet systématique du produit incriminé de l'ensemble de considération. En effet, plus la durée de la rumination est importante, plus il est facile 
d'identifier l'objet de cette même rumination grâce aux associations mentales dont elle a bénéficié (Martin et Tesser, 1989). La rumination pourrait également avoir un effet de feedback sur l'insatisfaction du consommateur et les réactions affectives post-achat, du à son effet exacerbateur (Lyubomirsky et Nolen-Hoeksema, 1995 ; Nolen-Hoeksema et Morrow, 1993). Elle serait alors susceptible d'entraîner une insatisfaction disproportionnée voire de la rancune de la part des consommateurs une fois l'achat effectué. Enfin, la rumination augmentant le temps nécessaire à la prise de décision (Nolen-Hoeksema, 2000b), elle est susceptible de favoriser le report d'achat. La Figure 2 illustre son rôle modérateur.

\section{Figure 2 : le rôle modérateur de la rumination}

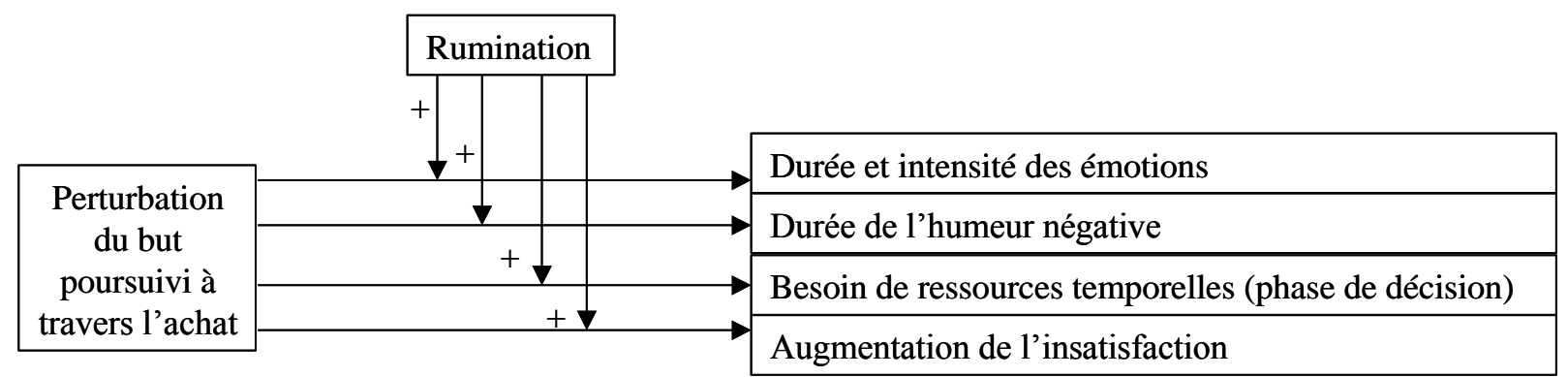

La rumination est susceptible d'intervenir de différentes manières dans le processus d'achat. Avant l'achat, elle met en évidence des conséquences inattendues comme une baisse de l'attention et de la motivation, affectant les règles de décision et fragilisant le lien entre intention et comportement. Après l'achat, la rumination est susceptible de prolonger les émotions négatives et d'accroître l'insatisfaction. Ces conséquences permettent de prêter à la rumination la capacité à modifier les effets cognitifs reconnus des émotions négatives comme l'augmentation de la vigilance et de l'attention aux informations externes (Forgas, 2002). Par ailleurs, cet article met en évidence des concepts qui font l'objet de recherches en comportement du consommateur : le regret (Gilovitch et Medvec, 1995 ; Tsiros et Mittal, 2000), qui intervient généralement suite à un achat insatisfaisant, et particulièrement susceptible d'être prolongé et intensifié par la rumination ; la procrastination, apparaissant ici 
comme une conséquence majeure de la rumination en phase de décision d'achat et partageant avec celle-ci un lien avec «l'orientation-attente » (Beswick et Mann, 1994). Une suite logique de ce premier travail de recherche serait donc d'articuler ces variables entre elles et les présenter dans un cadre conceptuel destiné à mieux comprendre les perturbations du processus de décision d'achat.

\section{Rumination et tendance à ruminer du consommateur}

Si nous avons présenté la rumination comme un phénomène susceptible de concerner chaque individu, dans de nombreux travaux de recherche en psychologie, la rumination est étudiée sous un angle dispositionnel (Roger et al., 2001; Scott et Mc Intosh, 1999; Sukhodolsky, Golub et Crowell, 2001). Ainsi, tous les individus ne seraient pas égaux face à la rumination. Certains individus présenteraient une véritable tendance à entrer plus facilement dans un cycle de rumination que d'autres. La question qui se pose alors est de savoir si la tendance à ruminer est en fait liée à une autre dimension stable de la personnalité, comme la dimension neuroticisme du modèle en cinq facteurs (que nous avons évoqué comme facteur influençant la rumination) ou s'il est possible de l'envisager à part entière comme un trait stable de la personnalité, par opposition à un état lié à des facteurs situationnels. Cette dernière hypothèse, évoquée par plusieurs chercheurs, suppose qu'il faille chercher la présence ou l'absence d'une tendance à ruminer chez le consommateur, ce qui implique la construction d'une échelle de mesure psychométrique. Plusieurs échelles de mesure de la rumination ont été développées à ce jour par les psychologues, mais exclusivement dans le cadre d'études sur les désordres mentaux. La littérature a montré que la rumination interagit avec de nombreux facteurs individuels, qui permettent de dresser un premier portrait robot du « ruminateur ». Celui-ci, outre sa jeunesse et son inexpérience, serait plus souvent une femme et aurait a priori le profil suivant : une tendance plus forte que la 
moyenne à persister dans une action; une tendance à s'attribuer la responsabilité en cas d'échec; une plutôt faible estime de soi ; une tendance à regretter ses décisions, à être pessimiste et à se situer davantage dans la réflexion que dans l'action ; enfin il éprouverait des difficultés à communiquer. On pourrait s'intéresser à compléter cette connaissance du ruminateur et s'intéresser à la pertinence d'un tel profil de consommateur. Il pourrait s'agir par exemple, dans un premier temps, d'interroger des consommateurs sur des expériences d'achat sources d'insatisfaction ou perçues comme risquées les ayant conduit à ressasser des pensées négatives sur une période de temps importante. L'objectif d'une telle recherche, outre la proposition d'un outil de mesure adapté à une utilisation dans le cadre de la consommation, pourrait être d'établir des liens entre la «Tendance à Ruminer» et d'autres variables individuelles étudiées en comportement du consommateur, comme la tendance à procrastiner (Darpy, 2002) ou la tendance à regretter (Delacroix, 2003), pour laquelle cet auteur a pu établir un lien significatif avec la rumination. Envisager la rumination sous cet angle permettra d'en affiner notre connaissance, de proposer un outil de mesure utilisable dans le contexte de la consommation et de dresser un profil psychologique et démographique du consommateur ayant tendance à ruminer, qu'il s'agira ensuite de comparer aux profils récemment dressés en comportement du consommateur, afin de mieux comprendre certains dysfonctionnements dans le processus de décision d'achat.

\section{La communication comme premier cadre de recherche sur la rumination positive?}

La rumination n'a fait l'objet, à la connaissance des auteurs, d'aucune recherche à propos de pensées positives récurrentes. Cette absence de travaux ne signifie pas pour autant que la rumination positive n'existe pas. Martin et Tesser (1996a) en donnent de nombreux exemples (comme l'anticipation positive et répétée d'un but à atteindre), d'autres évoquent leur possibilité d'occurrence (Koole et al., 1999; Rimé et al., 1992 ; Wänke et Schmid, 
1996). Il n'existe donc pas de raisons théoriques permettant de les écarter. Dans une perspective marketing, la rumination positive semble constituer une piste intéressante dans le cadre de la communication. En effet, de nombreuses campagnes publicitaires reposent sur l'incomplétude et la répétition d'un message et sont destinées à maintenir le consommateur en haleine (le rasoir Gillette Mach 3, le film Star Wars etc.) et font donc écho aux différentes dimensions de la rumination. Dans ce contexte, les messages pourraient axer leurs discours vers la capacité de la marque à permettre au consommateur d'atteindre un but. Par ailleurs, l'étude de la rumination positive pourrait s'avérer prometteuse dans le cadre des processus de mémorisation de la marque basés sur les réseaux sémantiques (Collins et Loftus, 1975). En raison des nombreuses répétitions mentales qu'elle entraîne, la rumination positive pourrait permettre de renforcer les connexions en mémoire entre une expérience positive (par exemple, l'achat d'un produit contribuant à l'atteinte de buts individuels) et la marque. Enfin, au-delà du champ de la communication, il serait intéressant de comprendre de quelle manière la rumination peut influencer les réactions affectives positives en les intensifiant et en modifiant leurs conséquences comportementales classiques, au premier rang desquelles la résolution de problème, la prise de risque et la recherche de variété (Isen, 2001). Ce terrain d'application pourrait permettre de mieux comprendre les mécanismes de la rumination positive, et d'en mesurer ses conséquences pratiques notamment dans le champ de la communication.

\section{CONCLUSION}

En envisageant les pensées ruminatives comme un phénomène général, détaché du contexte exclusif des désordres mentaux, et en l'inscrivant dans le champ motivationnel Martin et Tesser (1989) ont ouvert un nouveau champ de réflexion. A la connaissance des auteurs, ce champ nouveau n'a jamais fait l'objet de travaux en comportement du 
consommateur. Cependant, les caractéristiques proposées par la littérature et particulièrement par la «théorie de la rumination» (centralité des buts, dimension temporelle ou valence) permettent d'ancrer la rumination dans le processus d'achat, d'envisager de liens entre des variables étudiées séparément jusqu'à présent (notamment le regret et la procrastination) et de suggérer des pistes pour expliquer certaines perturbations du processus de décision d'achat. Ainsi, mieux comprendre la rumination peut être bénéfique au manager dans le cadre de la relation client. En effet, la rumination perturbant considérablement les capacités cognitives du consommateur, elle le pousse à des comportements «illogiques », qui pourraient s'expliquer par une remise en cause de certains liens classiques entre variables (satisfaction et ré achat par exemple) dans des contextes favorisant l'apparition de pensées ruminatives. Notre réflexion suggère également la possibilité d'une tendance individuelle du consommateur à ruminer, donc l'intérêt de développer et valider une échelle de mesure de la tendance à ruminer dans un contexte de consommation. Enfin, cet article souligne l'existence théorique d'une rumination positive, dont la compréhension des mécanismes pourrait entre autres contribuer à expliquer le fonctionnement de certaines techniques de communication. 


\section{RÉFÉRENCES BIBLIOGRAPHIQUES}

Alderson W (1957), Marketing behavior and executive action, Homewood, IL:Richard D. Irwin.

Austin J.T. and Vancouver J. B. (1996), Goal constructs in psychology : structure, process, and content, Psychological Bulletin, 120, 3, 338-375.

Bagozzi R.P. (1993), In the neglect of volition in consumer research : a critique and proposal, Psychology \& Marketing, 10, 215-237.

Bagozzi R.P. et Warshaw P.R. (1990), Trying to consume, Journal of Consumer Research, 17, September, 127-140.

Baumeister R.F. (1991), Meanings of life, New York : Guilford Press.

Baumeister R.F., Bratslavsky E., Finkenauer C. et Vohs K.D. (2001), Bad is stronger than good, Review of General Psychology, 5, 323-370.

Beswick G. et Mann L. (1994), State orientation and procrastination, in J. Kuhl \& J. Beckmann (Eds.), Volition and Personality, Göttingen : Hogrefe \& Huber Publishers, 391396.

Burnett M.S. et Lunsford D.A. (1994), Conceptualizing guilt in the consumer decisionmaking process, Journal of Consumer Marketing, 11, 3, 33-43. 
Bushman B. J. (2002), Does venting anger feed or extinguish the flame? Catharsis, rumination, distraction, anger, and aggressive responding, Personality and Social Psychology Bulletin, 28, 724-731.

Carver C.S. et Scheier M.F. (1981), Attention and self regulation : a control theory approach to human behavior, New York : Springer-Verlag.

Carver C.S. et Scheier M.F. (2000), Structure of behavioral self-regulation, in M. Boekaerts, P. R. Pintrich, \& M. Zeidner (Eds.), Handbook of self-regulation San Dieg : Academic Press, 13-39.

Collins A.M. et Loftus E.S. (1975), A spreading-activation theory of semantic processing, Psychological review, 82, 6, 407-428.

Cox D.F. (1967), Risk taking and information handling in consumer behaviour, Harvard University Press, Boston, MA.

Darpy D. (2002), Le report d'achat expliqué par le trait de procrastination et le potentiel de procrastination, Recherche et Applications en Marketing, 17, 2, 1-21.

Davis R. et Nolen-Hoeksema S. (2000), Cognitive inflexibility among ruminators and nonruminators, Cognitive Therapy and Research, 24, 6, 699-711. 
Delacroix E. (2003), La tendance à regretter du consommateur, Thèse de doctorat en Sciences de Gestion, Université de Paris-Dauphine.

Di Paula A. et Campbell J.D. (2002), Self-esteem and persistence in the face of failure, Journal of Personality and Social Psychology, 83, 3, Sept., 711-724.

Ekman P. (1999), Basic Emotions, in T. Dalgleish and M. Power (Eds.). Handbook of Cognition and Emotion. Sussex, U.K. : John Wiley \& Sons, Ltd, 45-60.

Forgas, J. P. (2002), Feeling and doing: Affective influences on interpersonal behavior, Psychological Inquiry, 13, pp. 1-28.

Frijda N. (1988), The law of emotion, American Psychologist, 42, 377-399.

Garbarino E.C. et Edell J.A. (1997), Cognitive effort, affect, and choice, Journal of Consumer Research, 24, 2, September, 147-158.

Gilovich T. et Medvec V.H. (1995), The experience of regret: what, when, and why?, Psychological Review, 102, 2, 379-395.

Gold D.B. et Wegner D.M (1995), Origins of ruminative thought : Trauma, incompleteness, nondisclosure, and suppression, Journal of Applied Social Psychology, 25, 14, 1245-1261. 
Gollwitzer P. (1990), Action phases and mind-sets, in E.T. Higgins \& R.M. Sorrentino (Eds), Handbook of motivation and cognition : foundations of social behaviours, New York : Guilford Press, 2, 53-92.

Heckhausen H. ( 1991), Motivation and action, New York : Springer

Heim E. (1995), Coping-based intervention strategies, Patient Education and Counseling, 26, $145-151$

Horowitz, M.J. (1976), Stress response syndromes, New York, NY: Jason Aronson.

Huffman C., Rattneshwar S. et Mick D.G. (2000), Consumer goal structures and goal determination process : an integrative framework, in S. Rattneshwar, D.G. Mick et C. Huffman (eds), The Why of consumption : contemporary perspectives on consumer motives, London : Routledge, 9-35.

Isen A. M. (2001), An influence of positive affect on decision making in complex situations: theoretical issues with practical implications, Journal of Consumer Psychology 11, 2, 75-85.

Janoff-Bulman R. (1992), Shattered assumption : Towards a new psychology of traumas, New York, Free Press.

King L. et Pennebaker J. (1996), Thinking about goals, glue and the meaning of life, in Wyer R. eds, Ruminative thoughts, Advances in Social Cognition, Hillsdale : Erlbaum, 9, 97-106. 
Koole S.L., Smeets K., Van Knippenberg A., Dijksterhuis A. (1999). The cessation of rumination through self-affirmation, Journal of Personality and Social Psychology, 77, 1, $111-125$.

Kuhl J. (1994), A theory of action and state orientation, in J. Kuhl and J. Beckmann (Eds.), Volition and personality, Göttingen : Hogrefe \& Huber Publishers, 9-46.

Lazarus R. S., et Folkman, S. (1984), Stress, appraisal, and coping, New York: Springer.

Luce M.F, Bettman J.R. et Payne J.R. (1999), Emotional trade-off, difficulty and choice, Journal of Marketing Research, 36, May, 143-159.

Luminet, O. (2002), Psychologie des émotions. Confrontation et évitement, De Boeck Université, Bruxelles.

Luminet O., Zech E., Rimé B., et Wagner H.L. (2000), Predicting cognitive and social consequences of emotional episodes: The contribution of emotional intensity, the Five Factor Model and alexithymia, Journal of Research in Personality, 34 , 471-497.

Lyubomirsky S. et Nolen-Hoeksema S. (1995), Effects of self-focused rumination on negative thinking and interpersonal problem-solving, Journal of Personality and Social Psychology, 69, 176-190. 
Lyubomirsky S., Tucker K.L, Caldwell N.D et Berg K. (1999), Why ruminators are poor problems solvers : Clues from the phenomenology of dysphoric rumination, Journal of Personality and Social Psychology, 77, 5, 1041-1060.

Lyubomirsky S et Tkach C. (2003), The consequences of dysphoric rumination, in C.Papageorgiou \& A. Wells, Nature, Theory and treatment of negative thinking in depression, Chichester, England : John Wiley \& Sons, 21-41.

Martin L. et Tesser A. (1989), Toward a motivational and structural theory of ruminative thoughts, in Uleman J. et Bargh J. eds, Unintended thought, NY : The Guiliford Press, 306326.

Martin L. et Tesser A. (1996a), Some ruminative thoughts, in Wyer R. eds, Ruminative thoughts, Advances in Social Cognition, Hillsdale : Erlbaum, 9, 1-47.

Martin L. et Tesser A. (1996b), Clarifying our thoughts, in Wyer R. eds, Ruminative thoughts, Advances in Social Cognition, Hillsdale : Erlbaum, 9, 189-210.

Martin L., Tesser A. et McIntosh W.D. (1993), Wanting but not having : the effects of unattained goals on thoughts and feelings, in D.Wegner and J.Pennebaker (Eds), Handbook of Mental Control, Englewood Cliffs, NJ : Prentice Hall, 552-572.

Mc Rae R.R. et John O.P. (1992), An introduction to the five-factor model and its applications, Journal of Personality, 60, 175-215. 
Mellings T. et Alden L. (2000), Cognitive process in social anxiety : the effect of self-focus, rumination and anticipatory processing, Behaviour Research and Therapy, 38, 243-257.

Nolen-Hoeksema S. (1996), Chewing the cud and other ruminations, in Wyer R. eds, Ruminative thoughts, Advances in Social Cognition, Hillsdale : Erlbaum, 9, 135-144.

Nolen-Hoeksema S. (2000a), The role of rumination in depressive disorders and mixed anxiety/depressive symptoms, Journal of Abnormal Psychology, 109, 504 - 511.

Nolen-Hoeksema S. (2000b), Further evidence for the role of psychosocial factors in depression chronicity, Clinical Psychology: Science and Practice, 7, 224-227.

Nolen-Hoeksema S. (2005), Gender and Self-Regulation, in R.F. Baumeister \& K.D. Vohs (Eds.), Handbook of self-regulation: Research, theory, and application, New York : Guilford, $411-421$.

Nolen-Hoeksema S. et Morrow J. (1991), A prospective study of depression and posttraumatic stress symptoms after a natural disaster : the 1989 Loma Prieta earthquake, Journal of Personality and Social Psychology, 61, 115-121.

Nolen-Hoeksema S. et Morrow J. (1993), Effects of rumination and distraction on naturally-occurring depressed mood, Cognition and Emotion, 7, 561-570.

Nolen-Hoeksema S., Parker L.E. et Larson J. (1994), Ruminative coping with depressed mood following loss, Journal of Personality and Social Psychology, 67, 92-104. 
Rachman S.J. (1991), Fear and courage, $2^{\text {nd }}$ edition, New York: Freeman.

Rimé B., Philippot P., Boca S. et Mesquita B. (1992), Long-lasting cognitive and social consequences of emotion: social sharing and rumination, European Review of Social Psychology, 3, 225-257.

Roger D., Garcia de la Banda G., Lee H.S. et Olason D.T. (2001), A factor-analytic study of cross-cultural differences in emotional rumination and emotional inhibition, Personality and Individual Differences, 31, 2, 227-238.

Roger D. et Najarian B. (1989), The construction and validation of a new scale for measuring emotion control, Personality and Individual Differences, 10, 845-853.

Rusting C. L. et Nolen-Hoeksema S. (1998), Regulating responses to anger: Effects of rumination and distraction on angry mood, Journal of Personality and Social Psychology, 74, 790-803.

Scott V.B. et McIntosh W.D. (1999). The development of a trait measure of ruminative thought, Personality and Individual Differences, 26, 1045-1046.

Segerstrom S.C., Tsao J.C.I., Alden L.E. et Craske M.G. (2000), Worry and rumination : repetitive thoughts as a concomitant and predictor of negative mood, Cognitive Therapy and Research, 24, 6, 671-688. 
Siegle G. J., Steinhauer S.R., Carter C. S., Ramel W. et Thase M.E. (2003), Do the seconds turn into hours ? Relationships between sustained pupil dilation in response to emotional information and self-reported rumination, Cognitive Therapy and Research, 27, 365-383.

Silver R. L. et Wortman C. B. (1980). Coping with undesirable life events, in J.Garber \& M. E. P. Seligman (Eds.), Human helplessness : Theory and applications, New York: Academic Press, 279-340.

Sukhodolsky D., Golub A. et Cromwell E. (2001), Development of the anger rumination scale, Personality and Individual Differences, 31, 689-700.

Tait R. et Silver R. C. (1989). Coming to terms with major negative life events, in J.S. Uleman \& J. A. Bargh (Eds.), Unintended thought, New York: Guilford Press, 351-381.

Tangney J.P et Fischer K.W. (1995), Self-conscious emotions : The psychology of shame, guilt, embarrassment, and pride, New York: The Guilford Press.

Tracy J.L. et Robins R.W. (2004), Putting the Self Into Self-Conscious Emotions: A Theoretical Model, Psychological Inquiry, 15, 2, 103-125.

Trappnell P.D. et Campbell J.D. (1999), Private Self-Consciousness and the Five-Factor Model of Personality : Distinguishing Rumination From Reflection, Journal of Personality and Social Psychology, 76, 2, 284-304. 
Tsiros M. et Mittal V. (2000), Regret : a model of its antecedents and consequences in consumer decision making, Journal of Consumer Research, 26, march, 401-417.

Van Oppen P., Hoekstra R.J et Emmelkamp P.M.G. (1995), The structure of obsessivecompulsive symptoms, Behaviour Research Therapy, 33, 1, 15-23.

Van Rijsoort S., Emmelkamp P. et Vervaeke G. (2001), Assesment of worry and OCD : how are they related ?, Personality and Individual Differences, 31, 247-258.

Volle P. (1995), Le concept de risque perçu en psychologie du consommateur : antécédents et statut théorique, Recherche et Applications en Marketing, 10, 1, 39-56.

Verbeke W., Belschak F. et Bagozzi, R.P. (2004), The Adaptive Consequences of Pride in Personal Selling, Journal of the Academy of Marketing Science, 32, 4, 386-402.

Wänke R. et Schmid J. (1996), Rumination : when all else fails, in Wyer R. eds, Ruminative thoughts, Advances in Social Cognition, Hillsdale : Erlbaum, 9, 175-187.

Ward A., Lyubomirsky S., Sousa L. et Nolen-Hoeksema S. (2003), Can't quite commit : Rumination and uncertainty, Personality and Social Psychology Bulletin, 29, 1, January, 96107.

Watkins E. et Baracaia S. (2002), Rumination and social problem solving in depression, Behaviour Research and Therapy, 40, 10, 1179-1189. 
Watkins E. et Mason A. (2002), Mood as input and rumination, Personality and Individual Differences, 32, 577-587.

Wegner D.M. (1992), You can’t always think what you want : problems in the suppression of unwanted thoughts, Advances in Experimental Social Psychology, 25, 193-225.

Wegner D.M., Schneider D.J., Carter S.R. et White T.L (1987), Paradoxical effects of thoughts suppression, Journal of Personality and Social Psychology, 53, 5-13.

Wicklund R.A. et Braun O.L. (1987), Incompetence and the concern with human categories, Journal of Personality and Social Psychology, 53, 373-382.

Williams J. M. G., et Oaksford M. (1992). Cognitive science, anxiety, and depression: From experiments to connectionism, in Stein and Young (Eds.), Cognitive science and the clinical disorders, San Diego, CA: Academic Press, 129-150.

Zeigarnik, B. (1938), On finished and unfinished tasks, in W.D. Ellis (Ed and Trans), A source book of gestalt psychology, New York : Harcourt, Brace and World, 300-314. 